\title{
Champion de la prévention des risques infectieux dans les soins
}

Jean Martin

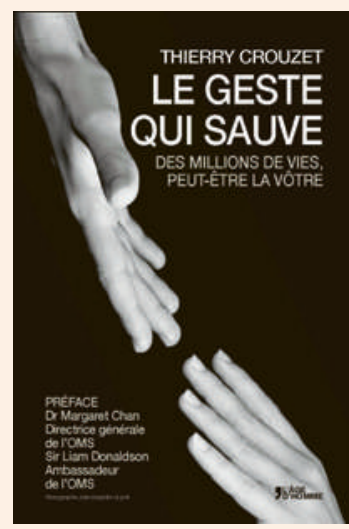

Thierry Crouzet Le geste qui sauve

Des millions de vies, peut-être la vôtre Lausanne/Paris: L'Age d'Homme; 2014 168 pages. 12 Euros. ISBN 978-2-8251-4400-8 (Traduit dans une demidouzaine de langues dont l'allemand et l'anglais)

* Qui au départ n'avaient rien auprès du milieu médical du prestige d'autres recherches à quelle gloire peut-on prétendre en s'intéressant au lavage des mains? Crouzet évoque la réaction d'un Doyen de la Faculté qui, tout en reconnaissant les mérites de D. Pittet, s'étonne qu'on puisse publier dans le Lancet avec de tels sujets.
Didier Pittet est professeur et directeur du Service de prévention et contrôle de l'infection aux Hôpitaux Universitaires de Genève. Il s'est acquis au cours des vingt dernières années une reconnaissance mondiale et a eu un impact majeur par ses travaux liés à la lutte contre la transmission d'infections dans les soins. Pour l'essentiel en promouvant le lavage fréquent des mains avec une solution hydro-alcoolique. Le médecin cantonal voisin que j'étais se souvient d'échos, à l'époque, sur ce jeune confrère dynamique qui aux HUG se passionnait pour l'hygiène hospitalière et entreprenait des études épidémiologiques, avec des composantes sociologiques et comportementales.

Un livre vient de lui être consacré. Brossant la fresque d'une vie d'engagements qui vont au-delà de la médecine. Enfance genevoise (né en 1957), études et formation médico-hospitalière sous l'égide du Prof. F. Waldvogel, choix de l'hygiène, séjour à Iowa City (1989-1992), développement de ses travaux scientifiques*, à la tête d'une équipe dévouée. Puis, tirant profit de la proximité, à Genève, de l'OMS, lancement avec elle du Programme «Clean Hands Save Lives» pour contrer la pandémie silencieuse des infections nosocomiales (responsables de 20000 à 50000 décès par jour dans le monde nous dit-on). Récit plein d'anecdotes petites ou grandes, tantôt drôles tantôt (ainsi dans les pays en développement) poignantes. Exemple politico-culturel: la nécessité de modifier la solution pour qu'elle devienne acceptable dans des pays islamiques qui craignent que l'alcool passe la peau.

On a comparé (ce que l'intéressé ne refuse pas) Pittet à Ignaz Semmelweis (1818-1865), le gynécologue qui à Vienne mit en évidence la transmission de la fièvre puerpérale d'une femme à l'autre par les médecins (mais qui n'a pas su convaincre, en a souffert et est mort misérablement). Il y a une telle dimension chez Didier Pittet, un combat pour une idée juste aux effets positifs majeurs. Thierry Crouzet met en évidence les différences qui ont fait le succès cette fois. Semmelweis a rencontré de vives résistances et/ mais s'est montré autoritaire, parfois insultant, suscitant des conflits qui l'ont mené à sa perte. Didier Pittet a toujours estimé qu'il fallait expliquer, démontrer et convaincre, sans vouloir imposer. Entraînant les gens avec lui, boy-scout, dit une collaboratrice. Un modèle d'approche multimodale; utilisant aussi la force institutionnelle, soignant la communication avec l'aide du dessinateur Pécub. Engagement constant, dans la durée. On peut parler de champion (titre du présent billet) aux deux sens du terme: de celui qui atteint des objectifs et gagne, et du défenseur d'une cause dans un sens d' «advocacy».

L'ouvrage bénéficie d'une préface de Margaret Chan, directrice générale de l'OMS, et de Sir Liam Donaldson, qui relèvent que le programme OMS «Un soin propre est un soin plus sûr» (Clean Care is Safer Care), qui doit beaucoup à D. Pittet, a été le premier défi global pour la sécurité des patients, lancé en 2002. «Le succès d'un tel programme implique une vision porteuse. Ce livre réhabilite les valeurs de l'hygiène si fermement démontrées par Florence Nightingale, il montre comment une mobilisation mondiale est possible. Il raconte l'histoire d'un leader capable de concrétiser son rêve de sauver des vies grâce à l'hygiène des mains [...]. Peu de gens ont entendu son nom mais beaucoup lui doivent leur santé et leur vie».

Thierry Crouzet souligne aussi comment D. Pittet n'a à aucun moment cherché à faire de l'argent avec le développement de sa solution hydro-alcoolique et du «Geneva Model» d'hygiène des mains aujourd'hui largement appliqué dans le monde - estimant qu'il a renoncé ainsi à amasser une fortune. Il y a dans cette aventure, dit-il, «la promesse de basculer d'une économie de prédation à une économie de paix». Intéressant changement de registre dans le propos, espoir de modèles alternatifs du vivre ensemble! Le fait est que, s'agissant d'améliorer la santé, ici et ailleurs, c'est un signe fort que d'accepter de mettre à disposition gratuitement les découvertes et développements que l'on a permis. On souhaiterait voir ce genre de service à la communauté mondiale aussi chez les entreprises produisant des médicaments ou des équipements et matériels médicaux. Etant admis que la gratuité généralisée est un objectif qui ne sera pas atteint, au moins attendrait-on moins d'exigences quant aux retours sur investissement - et parfois une vraie action philanthropique. Un article récent dénomme D. Pittet «Chevalier du don», le disant milliardaire raté - qui a refusé de le devenir [1]. Je ne sais si l'intéressé est à l'aise avec tous les honneurs (y compris une haute décoration britannique) et compliments qui lui sont adressés, mais «Le geste qui sauve» inclut des faits qui méritent d'être connus, débattus et enseignés.

1 Nikolic D. Chevalier du don. Le Temps (Genève), 30 avril 2014, p. 28. 